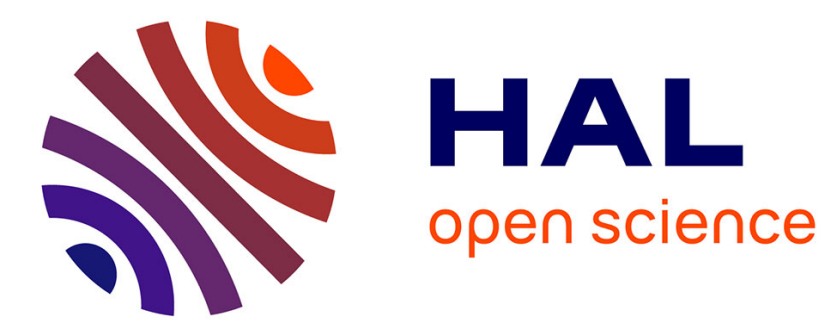

\title{
Observability and observer design for hybrid multicell choppers
}

Francisco Bejerano, Malek Ghanes, Jean-Pierre Barbot

\section{To cite this version:}

Francisco Bejerano, Malek Ghanes, Jean-Pierre Barbot. Observability and observer design for hybrid multicell choppers. International Journal of Control, 2010, 83 (3), 10.1080/00207170903334821 . hal00640515

\section{HAL Id: hal-00640515 https://hal.inria.fr/hal-00640515}

Submitted on 12 Nov 2011

HAL is a multi-disciplinary open access archive for the deposit and dissemination of scientific research documents, whether they are published or not. The documents may come from teaching and research institutions in France or abroad, or from public or private research centers.
L'archive ouverte pluridisciplinaire HAL, est destinée au dépôt et à la diffusion de documents scientifiques de niveau recherche, publiés ou non, émanant des établissements d'enseignement et de recherche français ou étrangers, des laboratoires publics ou privés. 


\title{
RESEARCH ARTICLE
}

\section{Observability and observer design for hybrid multicell choppers}

\author{
F.J. Bejarano ${ }^{a *}$, M. Ghanes ${ }^{b}$, and J-P. Barbot ${ }^{b \dagger}$ \\ ${ }^{a}$ Department of Control, Electrical Engineering Division,National Autonomous University of Mexico \\ (UNAM), México; ${ }^{b}$ Equipe Commande des Systèmes, ENSEA, France; ${ }^{\dagger}$ Equipe Projet ALIEN-INRIA \\ (Received 00 Month 200x; final version received 00 Month 200x)
}

\begin{abstract}
Multicell choppers are part of a class of hybrid systems in which the continuous state vector is always unobservable, in the sense that the observability matrix has never full rank. Due to their hybrid behavior, the recent concept of $Z\left(T_{N}\right)$-observability can be applied and analyzed in the context of multicell choppers; which allows to give conditions, in terms of the switching sequence, under which the voltage across each capacitor can be reconstructed, not instantly, but after some number of switchings. It is also considered the case when a DCmotor is coupled to the multicell chopper. In this case, it is shown, that under certain admissible assumptions, the voltages across the capacitors and the speed of the motor can be acceptably estimated. Two observers, one based on the super-twisting algorithm and the other one based on an adaptive approach, are designed. Additionally, we design an observer for the partial state observation. Simulations are given where the proposed observers are compared and their effectiveness is shown.
\end{abstract}

Keywords: hybrid systems; observability; multicell choppers

\section{Introduction}

Analysis, control, observation, and diagnosis of hybrid systems are nowadays of crucial interest in many industrial applications. This is mainly due to two reasons; the intensive computer use in the control of processes and the development of control theories dedicated to hybrid systems (Lygeros (2003)). On the other hand, the power electronics (Erickson (2001)) has had important technological developments, with many of them presenting a hybrid dynamics. An example is the multicell chopper which works perfectly for showing a kind of device that can be considered as a hybrid system. The multicell choppers are build based upon a series of elementary cells of commutation. This structure, appeared at the beginning of the 90's (Fadel and Meynard (1996)), makes it possible to share the constraints in tension and it also improves the harmonic contents of the wave forms (Donzel (2000)). From a practical point of view, the series of a multicell chopper, designed by the LEEI (Toulouse-France) (Bensaid and Fadel (2001)), leads to a safe series association of components working in a switching mode. This new structure combines additional benefits: attenuation of the voltage jump and modularity of the topologies. All these qualities make to this new topology very attractive in many industrial application. For instance, GEC/ACEC implements this proposal to realize the input chopper which supplies their "T13" locomotives in power. Three-phase inverters called "symphony" developed by Alstom for driving electric motors are based on the same principle too. Various research directions were developed in the literature to benefit as much as possible from the large potential of the multicell structure. The control inserted by the switches allows one to cancel the harmonics at the switching frequency and to reduce the ripple of the chopped voltage. However, these properties are lost if the voltages of these capacitors drift. Therefore, it is advisable to measure these voltages. An option for such

\footnotetext{
${ }^{*}$ This work was supported by Bourse region IDF. Corresponding author. Email: javbejarano@yahoo.com.mx 
a goal is the use of extra sensors; however, it increases considerable the cost of the overall system. Hence, the estimation of such voltages by means of an observer becomes an attractive economical option.

Several approaches have been considered to develop new methods of control and observation of the multicell chopper. Initially, models have been developed to describe their instantaneous (Donzel (2000)), harmonic (Fadel and Meynard (1996)) or averaging (Bensaid and Fadel (2001)) behaviors. These various models were used for the development of control laws in open-loop (Tachon (1997)) and in closed loop (Bethoux and Barbot (2002)). However, current control algorithms do not take into account the fact that any power chopper is a hybrid system. Here, it is proposed to estimate the voltages of the capacitors using an instantaneous model describing the dynamical hybrid behavior of the multicell chopper.

In fact, at any time, the state vector of a multicell chopper is not observable, i.e. the observability matrix has never full column rank; however, under certain conditions, there exists a time sequence, related to the so-called hybrid time trajectory (Lygeros (2003)), after which one can observe (reconstruct) the entire state vector. Such conditions can be found by means of the recently suggested $Z\left(T_{N}\right)$-observability concept (Kang and Barbot (2007)). In this manuscript two observers are proposed, one based on the super-twisting algorithm (see, e.g., Levant (1996), Davila et al. (2005) and references therein) and the other based on an adaptive approach (Hammouri and De Leon (1990), Ghanes et al. (2006), and references therein). According to the concept of $Z\left(T_{N}\right)$ observability, both observers allow estimating the voltage across the capacitors and the speed of the DC-motor coupled to the multicell chopper using the measurable variables: the current load and the source's voltage.

\section{Recalls on $Z\left(T_{N}\right)$-Observability}

In a recent paper (Kang and Barbot (2007)) the concept of $Z$-Observability was introduced in order to consider at the same time the observability, identifiability, and left invertibility problems. In the same way the $Z\left(T_{N}\right)$-Observability concept was also introduced in order to consider the observability of some particular class of hybrid systems. As the multicell chopper is included in the class of hybrid systems concerned by the $Z\left(T_{N}\right)$-Observability, we first recall this concept. Let us consider the following class of switched systems:

$$
\begin{aligned}
\dot{\xi} & =f_{q}(t, \xi, u), \quad q \in Q, \xi \in \mathbb{R}^{n}, u \in \mathbb{R}^{m} \\
Y & =h_{q}(t, \xi, u)
\end{aligned}
$$

Where $Q$ is a finite index set, $f_{q}: \mathbb{R} \times \mathbb{R}^{n} \times \mathbb{R}^{m} \rightarrow \mathbb{R}^{n}$ is sufficiently smooth, the dwell time $\left[t_{i, 0}, t_{i, 1}\right]$ between two switchings of the structure is such that there exists a constant $\tau_{\min }>0$ so that $t_{i, 1}-t_{i, 0}>\tau_{\min }$ (this exclude Zeno phenomenon). The input $u(t)$ is assumed to be bounded and $C^{\infty}$ on the interval $\left[t_{i, 0}, t_{i, 1}\right)$.

For switched systems the observation concept is strongly linked to the dwell time and the sequence of switching; thus, it is important to recall (in our context) the following definition of hybrid time trajectory (Lygeros (2003)) (see also Goel et al. (2004)).

Definition 2.1: A hybrid time trajectory is a finite or infinite sequence of intervals $T_{N}=$ $\left\{\mathcal{I}_{i}\right\}_{i=0}^{N}$, such that

- $\mathcal{I}_{i}=\left[t_{i, 0}, t_{i, 1}\right)$, for all $0 \leq i<N$;

- For all $i<N, t_{i, 1}=t_{i+1,0}$;

- $t_{0,0}=t_{\text {ini }}$ and $t_{N, 1}=t_{\text {end }}$.

Moreover, it is defined $\left\langle T_{N}\right\rangle$ as the ordered list of $q$ associated to $T_{N}$ (i.e., $\left\{q^{0}, \ldots, q^{N}\right\}$ with $q^{i}$ equal to $q$ on the time interval $\mathcal{I}_{i}$ ). 
Now, it is possible to give the recently proposed concept of $Z\left(T_{N}\right)$-observability, which can be considered as an extension of the classical concept of observability:

Definition 2.2 (Kang and Barbot (2007):) A function $z=Z(t, \xi, u): \mathbb{R} \times \mathbb{R}^{n} \times \mathbb{R}^{m} \rightarrow \mathbb{R}^{n_{z}}$ is said to be $Z\left(T_{N}\right)$-observable in $U \subset \mathbb{R} \times \mathbb{R}^{n} \times \mathbb{R}^{m}$ with respect to the system 1 and the hybrid time trajectory $T_{N}$ and $\left\langle T_{N}\right\rangle$ if for any two trajectories, $\left(t, \xi^{i}(t), u^{i}(t)\right), i=1,2$, in $U$ defined on the interval $\left[t_{\text {ini }}, t_{\text {end }}\right]$, the equality

$$
h\left(t, \xi^{1}(t), u^{1}(t)\right)=h\left(t, \xi^{2}(t), u^{2}(t)\right), \quad \text { a.e. in }\left[t_{\text {ini }}, t_{\text {end }}\right]
$$

implies

$$
Z\left(t, \xi^{1}(t), u^{1}(t)\right)=Z\left(t, \xi^{2}(t), u^{2}(t)\right), \text { a.e. in }\left[t_{\text {ini }}, t_{\text {end }}\right]
$$

Suppose for any trajectory $(t, \xi(t), u(t))$ in $U$, there always exists an open set $U_{1} \subset U$ so that $(t, \xi(t), u(t))$ is contained in $U_{1}$ and $Z(t, x, u)$ is $Z\left(T_{N}\right)$-observable in $U_{1}$ with respect to the system 1 and the hybrid time trajectory $T_{N}$ and $\left\langle T_{N}\right\rangle$. Then, $z=Z(t, x, u)$ is said to be locally $Z\left(T_{N}\right)$-observable in $U$ with respect to the system 1 and the hybrid time trajectory $T_{N}$ and $\left\langle T_{N}\right\rangle$.

Lemma 2.3 (Kang and Barbot (2007):) Consider the system 1 and the hybrid time trajectory $T_{N}$ and $\left\langle T_{N}\right\rangle$. Let $U$ be an open set in time-state-control space. Suppose $Z(t, x(t), u(t))$ is always continuous under an admissible control input. Suppose there exist $N+1$ linear projections $\left\{P_{i}\right\}$ so that

(1) given any $0 \leq i \leq N, P_{i} Z(t, \xi, u)$ is $Z$-observable for $t \in\left[t_{i, 0}, t_{i, 1}\right)$ and $(t, \xi(t), u(t)) \in U$;

(2) $\operatorname{rank}\left[P_{0}^{T} \cdots P_{N}^{T}\right]=\operatorname{dim}(Z)=n_{z}$; and

(3) $\frac{\bar{P}_{i} Z(t, \xi(t), u(t))}{d t}=0$ for $t \in\left[t_{i, 0}, t_{i, 1}\right)$ and $(t, \xi(t), u(t)) \in U$, where $\left[\bar{P}_{i}^{T} P_{i}^{T}\right]$ has full rank in $\mathbb{R}^{n_{z} \times n_{z}}$.

Then, $z=Z(t, \xi, u)$ is $Z\left(T_{N}\right)$-observable in $U$ with respect to the system 1 and the hybrid time trajectory $T_{N}$ and $\left\langle T_{N}\right\rangle$.

Remark 1: The hybrid time trajectory $T_{N}$ and $\left\langle T_{N}\right\rangle$ affects the observability property in a similar way to an input. Therefore, in a future work it could be interesting to define a universal hybrid time trajectory as the time trajectory which preserves the observability (see Gauthier and Kupka (2001) for the definition of an universal input).

\section{Multicell chopper models}

This section introduces the models of a $p$-cells chopper on RL and DC motor loads considered along the paper.

\section{$3.1 \quad$ On RL Load}

The dynamic model of a $p$-cells chopper (Figure 1) is governed by the following state equations:

$$
\left\{\begin{aligned}
\dot{I}(t) & =-\frac{R}{L} I(t)+\frac{E}{L} u_{p}-\sum_{j=1}^{p-1} \frac{v_{c_{j}}(t)}{L}\left(u_{j+1}-u_{j}\right) \\
\dot{v}_{c_{j}}(t) & =\frac{I}{c_{j}}\left(u_{j+1}-u_{j}\right), \quad(j=1, \ldots, p-1) \\
y(t) & =I(t)
\end{aligned}\right.
$$

where $I$ defines the load current, $v_{c_{j}}$ is the voltage in the $j$-th capacitor and $E$ represents the source's voltage. The control $u_{j} \in\{0,1\}$ is given by the position of the upper switch on the $j$-th cell; $u_{j}=0$ means an open position of the upper switch and, in the same cell, a closed position 
of the lower switch. $R$ and $L$ represent the load resistance and inductance, respectively; both are supposed to be constant.

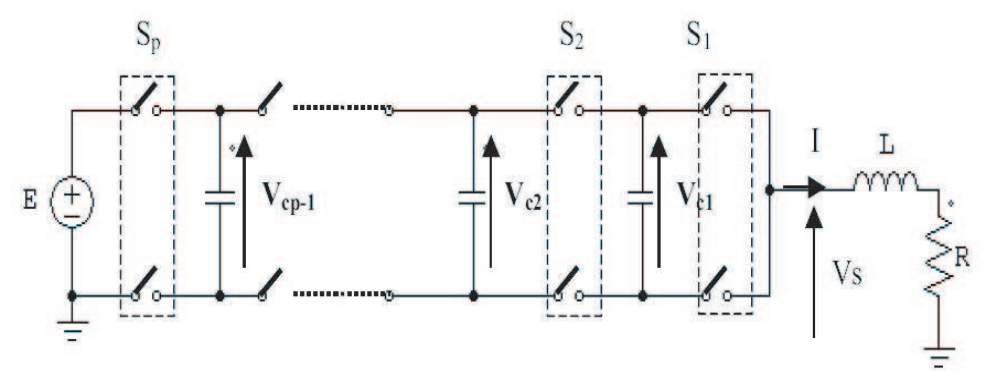

Figure 1. Multicell chopper on RL load.

\subsection{On DC motor load}

By considering a DC motor as a load, the dynamic model of a $p$-cells chopper (Figure 2) is described by the following equations

$$
\left\{\begin{aligned}
\dot{I}(t) & =-\frac{R}{L} I(t)+\frac{E}{L} u_{p}-\frac{k_{\mathrm{em}} \omega(t)}{L}-\sum_{j=1}^{p-1} \frac{v_{c_{j}}(t)}{L}\left(u_{j+1}-u_{j}\right) \\
\dot{v}_{c_{j}}(t) & =\frac{I}{c_{j}}\left(u_{j+1}-u_{j}\right), \quad(j=1, \ldots, p-1) \\
y(t) & =I(t)
\end{aligned}\right.
$$

where $\omega$ and the constant $k_{\mathrm{em}}$ represent the speed and the electromechanical coefficient of the DC-motor, respectively.

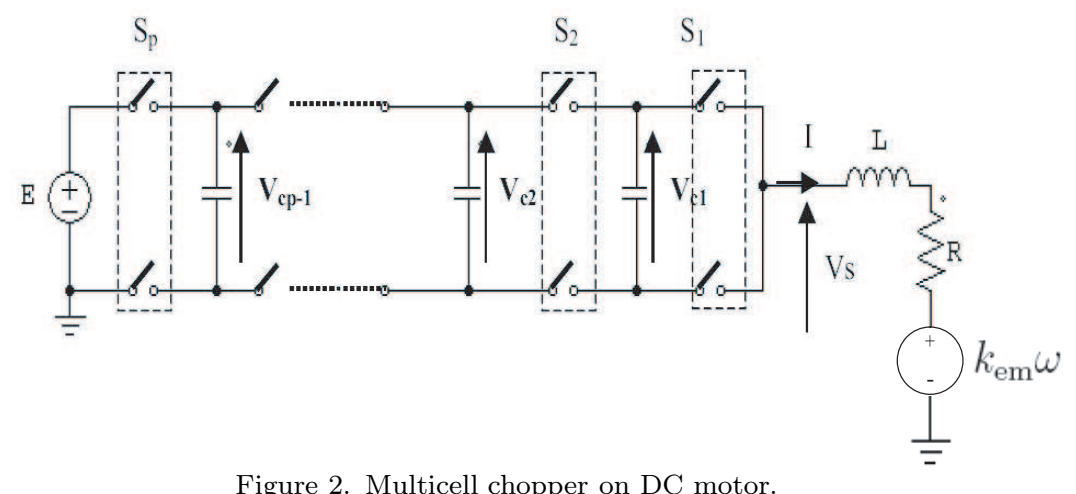

\subsection{Hybrid representation}

Taking the state vector as $x:=\left[\begin{array}{llll}I & v_{c_{1}} \ldots v_{c_{p-1}}\end{array}\right]^{T}$ and by the following definition of the vector $q \in\{-1,0,1\}^{p-1}$,

$$
\begin{aligned}
& q_{j}:=u_{j+1}-u_{j}, j=1, \ldots, p-1 \\
& q:=\left[q_{1} \cdots q_{p-1}\right] .
\end{aligned}
$$

The systems (2) and (3) can be represented by a hybrid matrix state equation, namely:

\section{On RL Load}

$$
\begin{aligned}
& \dot{x}=A(q) x+g\left(u_{p}\right) \\
& y=C x
\end{aligned}
$$


where

$$
\begin{aligned}
& A(q)=\left[\begin{array}{cccc}
-\frac{R}{L} & -\frac{q_{1}}{L} & \cdots & -\frac{q_{p-1}}{L} \\
\frac{q_{1}}{c_{1}} & 0 & \cdots & 0 \\
\vdots & \vdots & \ddots & \vdots \\
\frac{q_{p-1}}{c_{p-1}} & 0 & \cdots & 0
\end{array}\right], g\left(u_{p}\right)=\left[\begin{array}{c}
\frac{E}{L} u_{p} \\
0 \\
\vdots \\
0
\end{array}\right] \\
& C=\left[\begin{array}{llll}
1 & 0 & \cdots & 0
\end{array}\right]
\end{aligned}
$$

\section{On DC motor load}

$$
\begin{aligned}
& \dot{x}=A(q) x+g\left(u_{p}, \omega\right) \\
& y=C x
\end{aligned}
$$

where

$$
g\left(u_{p}, \omega\right)=\left[\begin{array}{c}
\frac{E}{L} u_{p}-\frac{k_{\mathrm{em}}}{L} \omega \\
0 \\
\vdots \\
0
\end{array}\right]
$$

\section{$4 \quad Z\left(T_{N}\right)$-observability for multicell chopper}

First let us consider the multicell chopper with a DC motor as a load (6). In order to observe the state and to determine the speed of the DC motor, the following assumption is necessary:

Assumption 4.1 The DC motor speed variation is considered negligible with respect to the electrical dynamic and the hybrid time trajectory (i.e. $\dot{\omega} \simeq 0$ ).

Lemma 4.2: Considering the system (6), and taking $z=\left[x^{T} \omega\right]^{T}$ the function $z(t)$ is $Z\left(T_{N}\right)$ observable, with respect to $T_{N}$ and $\left\langle T_{N}\right\rangle:=\left\{q^{0}, \ldots, q^{N}\right\}$, if assumption 4.1 is verified and the vectors $\left\{\left[q^{0} 1\right]^{T}, \ldots,\left[q^{N} 1\right]^{T}\right\}$ generate the space $\mathbb{R}^{p}$. Where $q^{i}=q$ on the interval $\mathcal{I}_{i}$, and $p$ is the number of cells.

Proof Let us take a basis $\left\{\left[q^{i_{1}} 1\right], \ldots,\left[q^{i_{p}} 1\right]\right\}$ of $\mathbb{R}^{p}$ from the vectors of $\left\langle T_{N}\right\rangle$ where $i_{1}<$ $\cdots<i_{p}$. Define $P_{i_{j}}=\left[\begin{array}{ccc}1 & 0 & 0 \\ 0 & q^{i_{j}} & 1\end{array}\right], P_{i_{j}} \in \mathbb{R}^{2 \times(p+1)}$, then $P_{i_{j}} z$ is observable on the interval $\mathcal{I}_{i_{j}}$. Let $F_{i_{j}}:=\left[F_{1, i_{j}} F_{2, i_{j}}\right]$ so that $q^{i_{j}} F_{i_{j}}^{T}=0$ and $\operatorname{rank}\left[P_{i_{j}}^{T} F_{i_{j}}^{T}\right]^{T}=p+1^{1}$. Thus by defining $G:=\operatorname{diag}\left(1, c_{1}, c_{2}, \ldots, c_{p-1}, 1\right)$, the identity

$$
\left[0 F_{i_{j}}\right] G \dot{z}=F_{1, i_{j}}\left(q^{i_{j}}\right)^{T} I=0
$$

\footnotetext{
${ }^{1}$ Notice that such matrix $F$ always does exist. Indeed, if $q^{i_{j}}=0$ then is enough to have rank $F_{1}=p-1$ and $F_{2}=0$. If $q^{i_{j}} \neq 0$, then $F=\left[\begin{array}{cc}\bar{F} & 0 \\ 0 & 1\end{array}\right]$ so that $q^{i_{j}} \bar{F}^{T}=0$ and $\operatorname{rank} \bar{F}=p-2$.
} 
holds on the interval $\mathcal{I}_{i_{j}}$. Since $\operatorname{rank}\left[P_{i_{j}}^{T}\left(F_{i_{j}} G\right)^{T}\right]=p+1$, by Lemma 2.3 the lemma is proven.

Define the vector $u^{i}=\left[u_{1}^{i} \cdots u_{p}^{i}\right]^{T}$ where $u_{j}^{i}=u_{j}$ on $\mathcal{I}_{i}(j=1, \ldots, p)$. The following corollary gives conditions for reconstruction of $\left[x^{T} \omega\right]^{T}$ in terms of a sequence of controls $u^{i}$.

Corollary 4.3: Considering (6) and taking $z=\left[x^{T} \omega\right]^{T}$, z is $Z\left(T_{N}\right)$-observable, with respect to $T_{N}$ and $\left\langle T_{N}\right\rangle:=\left\{q^{0}, \ldots, q^{N}\right\}$, if assumption 4.1 is verified and there exists a set of indexes $\left\{i_{1}, \ldots, i_{p}\right\}$ such that $\left\{\left[u^{i_{1} T} 1\right]^{T}, \ldots,\left[u^{i_{p} T} 1\right]^{T},\left[u^{o T} 0\right]^{T}\right\}$ is a basis of $\mathbb{R}^{p+1}$, where $u^{o} \in \mathbb{R}^{p}$ and $u_{j}^{o}=1(j=1, \ldots, p)$.

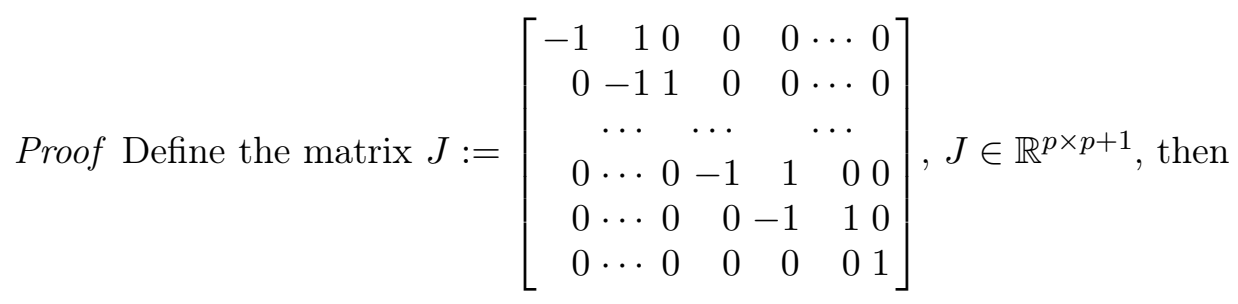

$$
J\left[\begin{array}{cccc}
u^{i_{1}} & \ldots & u^{i_{p}} & u^{o} \\
1 & \ldots & 1 & 0
\end{array}\right]=\left[\begin{array}{cccc}
q^{i_{1} T} & \ldots & q^{i_{p} T} & 0 \\
1 & \ldots & 1 & 0
\end{array}\right]
$$

which implies

$$
\operatorname{rank}\left\{\left(q^{i_{1}}, 1\right), \ldots,\left(q^{i_{p}}, 1\right)\right\}=\operatorname{rank} J=p
$$

Thus, the proof follows from (7) and lemma 4.2 .

Thus, we have given the conditions in terms of the control input $u$ under which the state vector can be reconstructed after a switching sequence.

Now, considering the multicell chopper with a $R L$ load given in (5), the following results are a direct consequence of Lemma (4.2) and Corollary (4.3).

Lemma 4.4: Considering the system (5) and taking $z=x$, the function $z(t)$ is $Z\left(T_{N}\right)$ observable, with respect to $T_{N}$ and $\left\langle T_{N}\right\rangle:=\left\{q^{0}, \ldots, q^{N}\right\}$, if the vectors $\left\{q^{0}, \ldots, q^{N}\right\}$ generate the space $\mathbb{R}^{p-1}$.

Proof The proof can be done by taking $P_{i_{j}}=\left[\begin{array}{cc}1 & 0 \\ 0 & q^{i_{j}}\end{array}\right]$ and following the proof of Lemma 4.2.

Corollary 4.5: Considering the system (5), $z=x$ is $Z\left(T_{N}\right)$-observable, with respect to $T_{N}$ and $\left\langle T_{N}\right\rangle:=\left\{q^{0}, \ldots, q^{N}\right\}$, if there exists a set of indexes $\left\{i_{1}, \ldots, i_{p-1}\right\}$ such that the set $\left\{u^{i_{1}}, \ldots, u^{i_{p-1}}, u^{o}\right\}$ generates the space $\mathbb{R}^{p}$.

\section{Design of the observers}

\subsection{Observation on $R L$ load}

Before describing the scheme of Super-Twisting Observer (STO) and the one of Adaptive Observer (AO), it must be assumed throughout this section that:

A1. The multicell chopper is decoupled from any motor, that is, the dynamics of the multicell chopper is governed by (2).

A2. There exists $T_{N}$ such that $z=x$ is $Z\left(T_{N}\right)$-observable with respect to (5). 
A3. There exists a constant $\tau>0$ so that the length $t_{i, 1}-t_{i, 0}>\tau$ for every interval $\mathcal{I}_{i}$.

Remark 2 : From the state equation (5), it is clear that on an interval of time $\mathcal{I}$ only the sum of voltages $\sum_{j=1}^{p-1} v_{c_{j}} q_{j}$ is observable. To overcome this restriction, Assumption A2 ensures that after the time interval $\mathcal{I}_{i_{p-1}}$, the information from the derivative of the load's current allows obtaining a set of $p-1$ linearly independent equations with respect to the voltages in the $p-1$ capacitors, which enable the estimation of the capacitor's voltages.

The next algorithm keeps the values of $q^{i_{j}}$ in such a way that after some sequence switches, ensured by Lemma 4.4 , the sequence of vectors $\left\{q^{i_{1}}, \ldots, q^{i_{p-1}}\right\}$ generates the space $\mathbb{R}^{p-1}$.

Algorithm 1: $\quad$ Let us define $H_{i} \in \mathbb{R}^{(p-1) \times(p-1)}$, in the interval $\mathcal{I}_{i}=\left[t_{i, 0}, t_{i, 1}\right)$ (for all $\left.0 \leq i<N\right)$ as a matrix formed by the following algorithm:

Step 0. $H_{-1}=0$.

Step 1. If $q^{i} \neq 0, H_{i, 1}=q^{i}$, where $H_{i, 1}$ is the first row of $H_{i}$, and set $j_{k}=i$. If $q=0, H_{i}=H_{i-1}$.

Step k. Let $H_{i, k}$ be the $k$-th row of $H_{i, k}$ for $k \geq 2$. Set $H_{i, k}=q^{j_{k}}$, where $j_{k}$ is the biggest index such that $j_{k-1}>j_{k}$ and the vectors $\left\{q^{j_{k}}, \ldots, q^{j_{1}}\right\}$ are linearly independent. If such an index $j_{k}$ does not exist, then $H_{i, k}=H_{i, k-1}$.

Let us define $H_{i}^{+}$as the pseudo-inverse of $H_{i}$. Below we will use the fact that for the case when $H_{i}$ is non-singular $H_{i}^{+} \equiv H_{i}^{-1}$.

With the matrix $H_{i}$ on the interval $\mathcal{I}_{i}$ defined with the previous algorithm, we are ready to introduce the observers: STO and AO.

\subsubsection{Description of the Super-Twisting Observer}

Taking into account the algorithm 2 and the definition of the indexes $j_{k}(k=1, \ldots, p-1)$, the STO is given, on the interval $\mathcal{I}_{i}(i=1,2, \ldots)$, by the following set of equations.

$$
\left\{\begin{array}{l}
\dot{x}_{\mathrm{a}}=-\frac{R}{L} I+\frac{E}{L} u_{p}-\frac{1}{L} q\left(\bar{v}_{\mathrm{c}}+\breve{v}_{\mathrm{c}}\right)+\sum_{j=1}^{p-1} \lambda\left|q_{j}\right|\left|I-x_{a}\right|^{1 / 2} \operatorname{sign}\left(I-x_{a}\right) \\
\dot{\bar{v}}_{\mathrm{c}_{j}}=\frac{I}{c_{j}} q_{j}, \bar{v}_{\mathrm{c}}^{T}=\left[\bar{v}_{\mathrm{c}_{1}} \cdots \bar{v}_{\mathrm{c}_{p-1}}\right] \\
\breve{v}_{\mathrm{c}_{j}}=-\alpha q_{j} \operatorname{sign}\left(I-x_{\mathrm{a}}\right), \breve{v}_{\mathrm{c}}^{T}=\left[\breve{v}_{\mathrm{c}_{1}} \cdots \breve{v}_{\mathrm{c}_{p-1}}\right] \\
\tilde{v}_{\mathrm{c}}^{T}=\left[q^{j_{1}} \breve{v}_{\mathrm{c}}^{j_{1}} \cdots q^{j_{p-1}} \breve{v}_{\mathrm{c}}^{j_{p-1}}\right] ; \breve{v}_{\mathrm{c}_{j}}^{j_{k}}(t)= \begin{cases}\breve{v}_{\mathrm{c}_{j}}, & t<t_{j_{k}, 1} \\
\breve{v}_{\mathrm{c}_{j}}\left(t_{j_{k}, 1}\right), t \geq t_{j_{k}, 1} & \\
\hat{\mathrm{v}}_{\mathrm{c}}(t)=\bar{v}_{\mathrm{c}}(t)+H_{i}^{+} \tilde{v}_{\mathrm{c}}(t), \hat{v}_{\mathrm{c}}^{T}=:\left[\hat{v}_{\mathrm{c}_{1}} \cdots\right.\end{cases}
\end{array}\right.
$$

with $\alpha$ and $\lambda(j=1, \ldots, p-1)$ satisfying

$$
\alpha>0, \lambda>\frac{(1+\theta)}{(1-\theta)} \sqrt{\frac{2 \alpha}{L}}, 0<\theta<1
$$

Defining $e_{1}=I-x_{\mathrm{a}}$ and $e_{\mathrm{c}_{j}}=v_{\mathrm{c}_{j}}-\hat{v}_{\mathrm{c}_{j}}(j=1, \ldots, p-1)$, we obtain 


$$
\begin{aligned}
\dot{e}_{1} & =-\frac{1}{L} q\left(v_{\mathrm{c}}-\bar{v}_{\mathrm{c}}+\breve{v}_{\mathrm{c}}\right)-\lambda\left|e_{1}\right|^{1 / 2} \operatorname{sign} e_{1} \sum_{j=1}^{p-1}\left|q_{j}\right| \\
\frac{d}{d t} q\left(v_{\mathrm{c}}-\bar{v}_{\mathrm{c}}+\breve{v}_{\mathrm{c}}\right) & =-(p-1) \alpha \operatorname{sign}\left(e_{1}\right)
\end{aligned}
$$

For $q$ constant, and $\alpha$ and $\lambda$ satisfying (9), there exists $T_{i}>t_{i, 0}$, such that

$$
e_{1}(t)=0, \dot{e}_{1}(t)=0 \text { for } t \geq T_{i}
$$

The equality (11) is assured only if $q$ stays fixed. Therefore, since $q$ is constant only on $\left[t_{i, 0}, t_{i, 1}\right)$, it must be ensured $T_{i}$ to be smaller than $t_{i, 1}$. From the proof of convergence given in Davila et al. (2005), one can obtain the time of converge to the sliding mode, that is,

$$
T_{i}-t_{i, 0} \leq \frac{1+\theta}{2 \theta \alpha} \sum_{j=1}^{p-1}\left|v_{\mathrm{c}_{j}}(0)-\bar{v}_{\mathrm{c}_{j}}(0)\right|
$$

Thus, choosing $\alpha$ enough big so that $T_{i}-t_{i, 0}<\tau$ and according to Assumption A3, one gets $T_{i}<t_{i, 1}$. Hence, from (11) and the equation for $\dot{e}_{1}$ in (10a), one deduce

$$
q\left(v_{\mathrm{c}}-\bar{v}_{\mathrm{c}}-\breve{v}_{\mathrm{c}}\right)=0 . \text { for } t_{i, 1} \geq t \geq T_{i}
$$

Now, with the observer already designed, we need to be sure that the observer trajectories converge to the original state trajectories.

Theorem 5.1: Under assumptions A1-A3, the identities

$$
\hat{v}_{\mathrm{c}_{i}} \equiv v_{\mathrm{c}_{i}}, i=1, \ldots, p-1
$$

are achieved, for all $t \geq T_{i_{p-1}}$, where $T_{i_{p-1}}$ is the reaching time to the $i_{p-1}$-th sliding mode.

Proof From Lemma 4.4, A1 implies that there exist $(p-1)$ indexes $i_{k}(k=1, \ldots, p-1)\left(i_{k+1}>i_{k}\right)$ so that $\left\{q^{i_{1}}, \ldots, q^{i_{p-1}}\right\}$ is a set of $(p-1)$ linearly independent vectors, then, from (13), the set of equalities (15) holds.

$$
\begin{gathered}
q^{i_{p-1}} \bar{v}_{\mathrm{c}}(t)+q^{i_{p-1}} \breve{v}_{\mathrm{c}}^{i_{p-1}}(t)=q^{i_{p-1}} v_{\mathrm{c}}(t), \text { for } t \in\left[T_{i_{p-1}}, t_{i_{p-1}, 1}\right) \\
\vdots \\
q^{i_{1}} \bar{v}_{\mathrm{c}}(t)+q^{i_{1}} \breve{v}_{\mathrm{c}}^{i_{1}}(t)=q^{i_{1}} v_{\mathrm{c}}(t), \text { for } t \in\left[T_{i_{1}}, t_{i_{1}, 1}\right)
\end{gathered}
$$

Since $\dot{v}_{\mathrm{c}}(t)-\dot{\bar{v}}_{\mathrm{c}}(t)=0$ for all $t \geq 0, q^{i_{k}} v_{\mathrm{c}}(t) \equiv q^{i_{k}} \bar{v}_{\mathrm{c}}(t)+q^{i_{k}} \breve{v}_{\mathrm{c}}^{i_{k}}\left(T_{i_{k}}\right)$ for all $t \geq T_{i_{k}}$. Obviously $\breve{v}_{\mathrm{c}}^{i_{k}}(t)$ stays constant on $\left[T_{i_{k}}, t_{i_{k}, 1}\right)$. Rearranging (15), into a matrix equation, we have

$$
\bar{v}_{\mathrm{c}}+H_{i}^{-1} \tilde{v}_{\mathrm{c}}=v_{\mathrm{c}} \text { for all } t \geq T_{i_{p-1}}
$$

A comparison between (16) and the formula that defines $\hat{v}_{\mathrm{c}}$ in (8) finishes the proof.

\subsubsection{Description of an Adaptive Observer}

Firstly, we give some preliminaries which will be considered in the sequel. 
5.1.2.1 Preliminaries. Let us first recall the result on state adaptive observer design (Besancon et al. (2006), Hammouri and De Leon (1990)), for so-called state-affine systems of the form

$$
\Sigma_{S A}:\left\{\begin{array}{l}
\dot{x}(t)=A(u(t)) x(t)+g(u(t), y(t)) \\
y(t)=C x(t)
\end{array}\right.
$$

where $x \in \mathbb{R}^{n_{x}}$ is the state vector of the system, $u \in \mathbb{R}^{n_{u}}$ is the control vector and $y \in \mathbb{R}^{n_{y}}$ is the output vector. The components of the matrix $A$ depends on the input. The vector field $g$ represents an input-output injection. The result can be summarized as follows.

First, it must be assumed that the input $u$ is persistently exciting, in the sense that there exist $\alpha, \beta, T>0$ and $t_{0}>0$ such that for all initial conditions $x_{0}$ the following inequalities are fulfilled:

$$
\alpha I \leq \int_{t}^{t+T} \Phi_{u, x_{0}}^{T}(\tau, t) C^{T} C \Phi_{u, x_{0}}(\tau, t) d \tau \leq \beta I
$$

where $\Phi_{u, x_{0}}$ denotes the transition matrix for system $\dot{x}=A(u) x, y=C x$. Then, an exponential observer for system (17) is given by

$$
O_{S A}:\left\{\begin{array}{l}
\dot{\hat{x}}(t)=A(u(t)) \hat{x}(t)+g(u(t), y(t))-P^{-1} C^{T}(\hat{y}(t)-y(t)) \\
\dot{P}(t)=-\theta P(t)-A^{T}(u(t)) P(t)-P(t) A(u(t))+2 C^{T} C \\
\hat{y}(t)=C \hat{x}(t)
\end{array}\right.
$$

for some positive constant $\theta$ sufficiently large and $\exists \theta_{0}>0$ such that for any symmetric positive definite matrix $P(0)$;

$$
\begin{gathered}
\forall \theta \geq \theta_{0} \exists \gamma>0, \delta>0, t_{0}>0: \forall t \geq t_{0}, \\
\gamma \mathbf{I} \leq P(t) \leq \delta \mathbf{I} .
\end{gathered}
$$

Defining the estimation error $\epsilon(t)=\hat{x}(t)-x(t)$, its dynamics is given by

$$
\dot{\epsilon}=\left(A(u(t))-P^{-1} C^{T} C\right) \epsilon(t)
$$

and consider the following candidate Lyapunov function $V(\epsilon(t))=\epsilon(t)^{T} P \epsilon(t)$. From (18), this Lyapunov function is well defined. Then, the following inequalities are satisfied $\bar{\alpha}\|\epsilon(t)\|^{2} \leq$ $V(\epsilon(t)) \leq \bar{\beta}\|\epsilon(t)\|^{2}$. Taking the time derivative of $V$ along the trajectory of the estimation error dynamics $\dot{\epsilon}=\left(A(u(t))-P^{-1} C^{T} C\right) \epsilon(t)$ we have

$$
\dot{V}(\epsilon(t)) \leq-\theta V(\epsilon(t))
$$

Then, the exponential convergence of the observer is proven.

5.1.2.2 Application to the multicell chopper. Consider the multicellular chopper model (5). As mentioned in remark 2 , on an interval of time $\mathcal{I}$ only the sum of voltages $q v_{\mathrm{c}}$ is observable. From this point on view let us define the quantity $b:=q v_{\mathrm{c}}$ as a state variable. Then, we can define the new state vector $X^{T}:=\left[\begin{array}{ll}I & b\end{array}\right]$ whose dynamics is governed, according with (5), by the following equations: 


$$
\begin{aligned}
& \dot{X}=A X+G(u, y) \\
& y=C X
\end{aligned}
$$

where

$$
\begin{aligned}
& A=\left[\begin{array}{cc}
0 & -\frac{1}{L} \\
0 & 0
\end{array}\right], G(u, y)=\left[\begin{array}{l}
\frac{E}{L} u_{p}-\frac{R}{L} I \\
\sum_{j=1}^{p-1} \frac{\left|q_{j}\right|}{c_{j}} I
\end{array}\right] \\
& C=\left[\begin{array}{ll}
1 & 0
\end{array}\right] .
\end{aligned}
$$

We can remark that (20) is on the form of (17) where the matrix $A(u)=A$ is constant.

Property 5.2 Due to $A(u)=A$, and $(A, C)$ is observable, the persistency excitation defined by (18) is verified for all time.

Then an A0 to estimate the capacitors voltages of the multicellular by using only the current measurement is described by following set of equations:

$$
\left\{\begin{array}{l}
\dot{Z}=A Z+G(u, y)+P^{-1} C^{T}(y-\hat{y}) \\
\dot{P}=-\rho P-A^{T} P-P A+2 C^{T} C \\
\hat{y}=C Z \\
Z^{T}=:[\hat{I} \hat{b}] \\
\dot{\bar{v}}_{c_{j}}=\frac{q_{j}}{c_{j}} I ; \quad \bar{v}_{\mathrm{c}}=\left[\bar{v}_{\mathrm{c}_{1}} \cdots \bar{v}_{\mathrm{c}_{p-1}}\right]^{T} \\
\Lambda=\hat{b}-q \bar{v}_{\mathrm{c}} \\
\left.\tilde{v}_{\mathrm{c}}=\left[\begin{array}{ll}
\Lambda^{j_{1}} \cdots \Lambda^{j_{p-1}}
\end{array}\right]^{T} ; \quad \Lambda^{j_{k}} \equiv \Lambda \text { on } \mathcal{I}_{j_{k}}=[t)=\Lambda_{j_{k}, 0}, t_{j_{k}, 1}\right) \\
\hat{v}_{\mathrm{c}}=\bar{v}_{\mathrm{c}}+H_{i}^{+} \tilde{v}_{\mathrm{c}} ; \quad \hat{v}_{\mathrm{c}}=\left[\hat{v}_{c_{1}} \cdots \hat{v}_{\mathrm{c}_{p-1}, 1}\right) \text { for } t \geq t_{j_{k}, 1}^{T}
\end{array}\right.
$$

with $\rho>0 . A, G(u, y)$ and $C$ are defined in $(20)$, and $H_{i}^{+}$is given by the algorithm 1.

5.1.2.3 Convergence analysis. Defining the estimation error $e=X-Z$ and consider the Lyapunov function candidate $V(e)=e^{T} P e$. By taking the time derivative of $V$ along the trajectory of the estimation error dynamics $\dot{e}=\left(A-P^{-1} C^{T} C\right) e$ we get $\dot{V}(e)=-\rho V(e)$. The solution of $V(e)$ is given by $V(e)=V(e(0)) e^{-\rho\left(t-t_{0}\right)}$. Then from property 5.2 , it is easy to see that

$$
\|e(t)\| \leq K\left\|e\left(t_{0}\right)\right\| e^{-\rho\left(t-t_{0}\right)}
$$

For $q$ constant and $\rho$ sufficiently large, it exists $T_{i}>t_{i, 0}$ such that

$$
\|e(t)\| \leq \gamma, \forall t \geq t_{i, 1}
$$

where $\gamma$ is an small constant error.

Since $q$ is constant on $t \in\left[t_{i, 0}, t_{i, 1}\right), T_{i}$ must be smaller that $t_{i, 1}$. From (22), the time of convergence is given by $T_{i}-t_{i, 0} \leq \frac{\log K\left\|e\left(t_{0}\right)\right\|-\log \gamma}{\rho}$. Hence, by choosing $\rho$ sufficiently large so that $T_{i}-t_{i, 0}<$ $\tau$ according to assumption A3, it follows that $T_{i}<t_{i, 1}$. Now from (23) and equation of $\Lambda$ in (21), 
the following is deduced

$$
\begin{aligned}
\Lambda^{j_{k}}+q \bar{v}_{\mathrm{c}} & =b+\delta^{j_{k}} \\
& =q v_{\mathrm{c}}+\delta^{j_{k}} \\
& =q v_{\mathrm{c}}+\delta^{j_{k}}
\end{aligned}
$$

where $\delta^{j_{k}}=\hat{b}-b$ on the interval $\mathcal{I}_{j_{k}}$, which, from (23), has a bounded norm: $\left\|\delta^{j_{k}}\right\|<\gamma$.

Theorem 5.3: $\quad$ Under assumptions A1-A3, by choosing $\rho$ sufficiently large, the identities

$$
\hat{v}_{\mathrm{c}}=v_{\mathrm{c}}+H_{i}^{+} \delta
$$

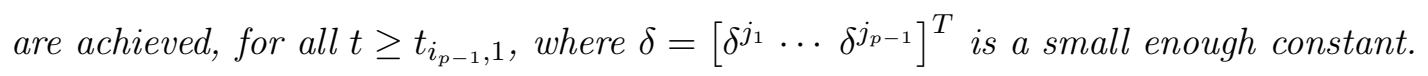

Proof From Lemma 4.4, A2 implies that there exist $p-1$ indexes $i_{k}(k=1, \ldots, p-1)\left(i_{k+1}>i_{k}\right)$ so that $\left\{q^{i_{1}}, \ldots, q^{i_{p-1}}\right\}$ is a set of $p-1$ linearly independent vectors, then, from (24), the set of equalities (26) holds.

$$
\begin{gathered}
\Lambda^{i_{p-1}}=q^{i_{p-1}}\left(v_{\mathrm{c}}-\bar{v}_{\mathrm{c}}\right)+\delta^{i_{p-1}}, t \geq t_{i_{p-1}, 1} \\
\vdots \\
\Lambda^{i_{1}}=q^{i_{1}}\left(v_{\mathrm{c}}-\bar{v}_{\mathrm{c}}\right)+\delta^{i_{1}}, t \geq t_{i_{1}, 1}
\end{gathered}
$$

Since $\dot{v}_{\mathrm{c}}(t)-\dot{\bar{v}}_{\mathrm{c}}(t)=0$ for all $t \geq 0$, the difference $v_{\mathrm{c}}-\bar{v}_{\mathrm{c}}$ stays constant. By rewritten (26), into a matrix equation, one has

$$
\tilde{v}_{\mathrm{c}}=\left[\begin{array}{c}
\Lambda_{i_{p-1}} \\
\vdots \\
\Lambda_{i_{1}}
\end{array}\right]=H_{i}\left(v_{\mathrm{c}}-\bar{v}_{\mathrm{c}}\right)+\delta \text { for all } t \geq t_{i_{p-1}, 1}
$$

Now, by replacing (27) in the equation of $\hat{v}_{\mathrm{c}}$ given by (21), one gets

$$
\begin{aligned}
\hat{v}_{\mathrm{c}} & =\bar{v}_{\mathrm{c}}+H_{i}^{+} H_{i}\left(v_{\mathrm{c}}-\bar{v}_{\mathrm{c}}\right)+H_{i}^{+} \delta \\
& =v_{\mathrm{c}}+H_{i}^{+} \delta, \quad \text { for all } t \geq t_{i_{p-1}, 1} .
\end{aligned}
$$

This end the proof.

\subsubsection{Simulations for a 3 cells chopper}

Let us consider a 3 cells chopper governed by the state equations (5) where $p=3$ and having the parameters $E=120 \mathrm{~V}, R=33 \Omega, L=50 \times 10^{-3} \mathrm{H}, c_{1}=c_{2}=40 \times 10^{-6} \mathrm{~F}$. Thus, by using the definition $q_{1}=u_{2}-u_{1}$ and $q_{2}=u_{3}-u_{2}$, one has:

$$
\left\{\begin{aligned}
\dot{I}(t) & =-6600 I(t)+2400 u_{3}(t)-20\left(q_{1} v_{\mathrm{c}_{1}}(t)+q_{2} v_{\mathrm{c}_{2}}(t)\right) \\
\dot{v}_{\mathrm{c}_{1}}(t) & =q_{1} \frac{10^{6}}{40} I(t) \\
\dot{v}_{\mathrm{c}_{2}}(t) & =q_{2} \frac{10^{6}}{40} I(t) \\
y(t) & =I(t)
\end{aligned}\right.
$$

The inputs $u_{j}, j=1, \ldots, 3$ are generated by a simple PWM where the triangular signal has a frequency of $700 \mathrm{~Hz}$. 
Remark 3: It should be noticed that with an usual (basic) converter at a frequency of $700 \mathrm{~Hz}$ it is not possible to consider a suitable PWM control. This is due to the voltage bandwidth of load (DC Motor), which is equal to $\frac{R}{L}=666 \mathrm{~Hz}$. Nevertheless, for the proposed 3 cells chopper with frequency of $700 \mathrm{~Hz}$, the voltage bandwidth of load is equal to $3 \times 700=2,1 \mathrm{kHz}$, which is much greater than $666 \mathrm{~Hz}$ and consequently this PWM can be applied.

The parameters of the STO are $\alpha=15000$ and $\lambda=5000$. The parameter $\rho$ of the AO is chosen as it follows, $\rho=1500$.

Figure 3 shows the voltage and its estimation in the first and second capacitors. It can be seen that the trajectories of both observers converge to the ones of the capacitors' voltages. Although in this figure the convergence of the STO is faster than the one of the AO, it must be said that, in theory, the rate of convergence in both observers can be modified by modifying their respective parameters.
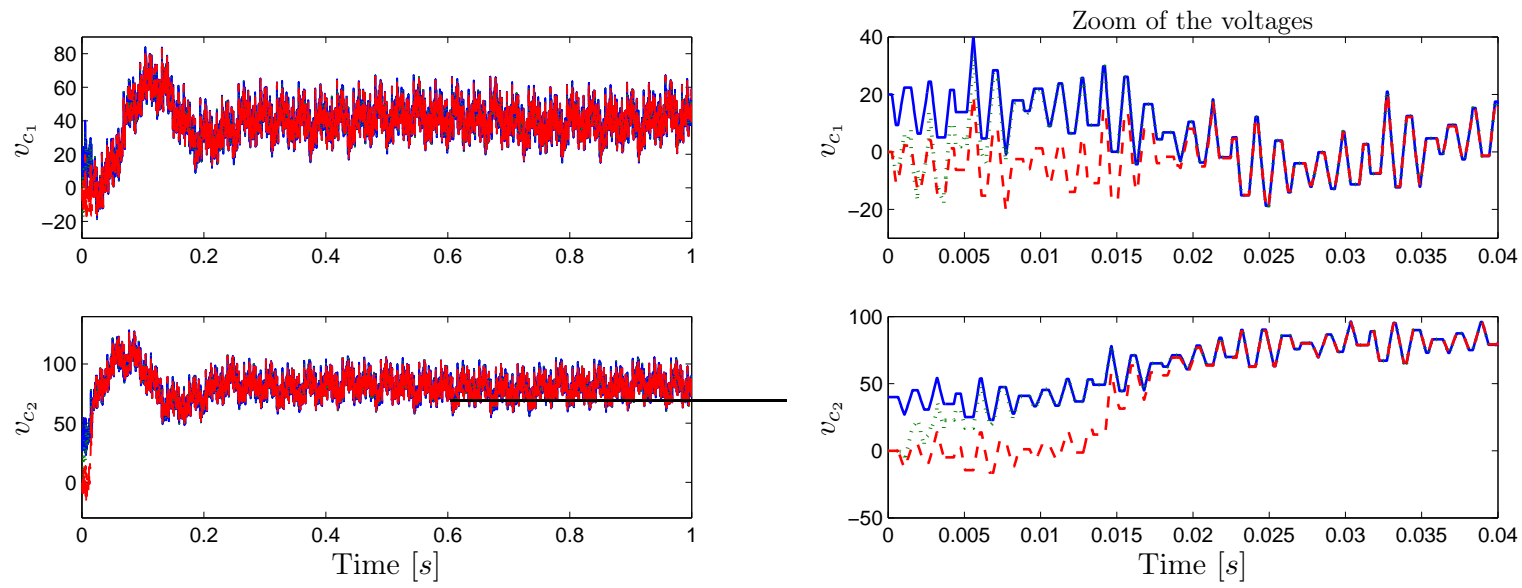

Figure 3. $v_{\mathrm{c}_{\mathrm{i}}}$ (solid) $(\mathrm{i}=1,2)$ and $\hat{v}_{\mathrm{C}_{\mathrm{i}}}$, STO (dot) and $\mathrm{AO}$ (dashed), for the case $k_{e m} \omega \equiv 0$.

\subsection{Observation on DC motor load}

Now it is assumed that a DC motor is coupled to the multicell chopper. Thus, in order to estimate the capacitor's voltage and the motor speed, it is assumed throughout this section that:

A4. For $\dot{\omega}=0$, there exists $T_{N}$ such that $z:=(x, \omega)$ is $Z\left(T_{N}\right)$-observable with respect to $(6)$.

A5. There exists a known constant $\omega^{+}$bounding $\dot{\omega}$, i.e., $|\dot{\omega}| \leq \omega^{+}$.

Before introducing the super twisting observer and the adaptive observer, let us design the matrix $H_{i}$ a little bit different than the one designed in the algorithm $\mathbf{1}$. Here, $H_{i} \in \mathbb{R}^{p \times p}$ is designed based on the following algorithm.

Algorithm 2: Construct $H_{i} \in \mathbb{R}^{p \times p}$, in the interval $\mathcal{I}_{i}=\left[t_{i, 0}, t_{i, 1}\right)$ (for all $0 \leq i<N$ ), by following the steps given below:

Step 1. $H_{i, 1}=\left[q^{i} k_{\mathrm{em}}\right]$, where $H_{i, 1}$ is the first row of $H_{i}$, and set $j_{1}=i$.

Step k. Let $H_{i, k}$ be the $k$-th row of $H_{i, k}$ for $k \geq 2$. Set $H_{i, k}=\left[q^{j_{k}} k_{\text {em }}\right]$, where $j_{k}$ is the biggest index such that $j_{k-1}>j_{k}$ and the vectors $\left\{\left(q^{j_{k}}, 1\right), \ldots,\left(q^{j_{1}}, 1\right)\right\}$ are linearly independent. If such an index $j_{k}$ does not exist, then $H_{i, k}=H_{i, k-1}$.

\subsubsection{Description of the Super-Twisting Observer}

With the matrix $H_{i}$ designed according to the algorithm $\mathbf{2}$, and the definition of the indexes $j_{k}$ given therein, the observer estimating the capacitor's voltages and the motor speed is designed 
as it follows,

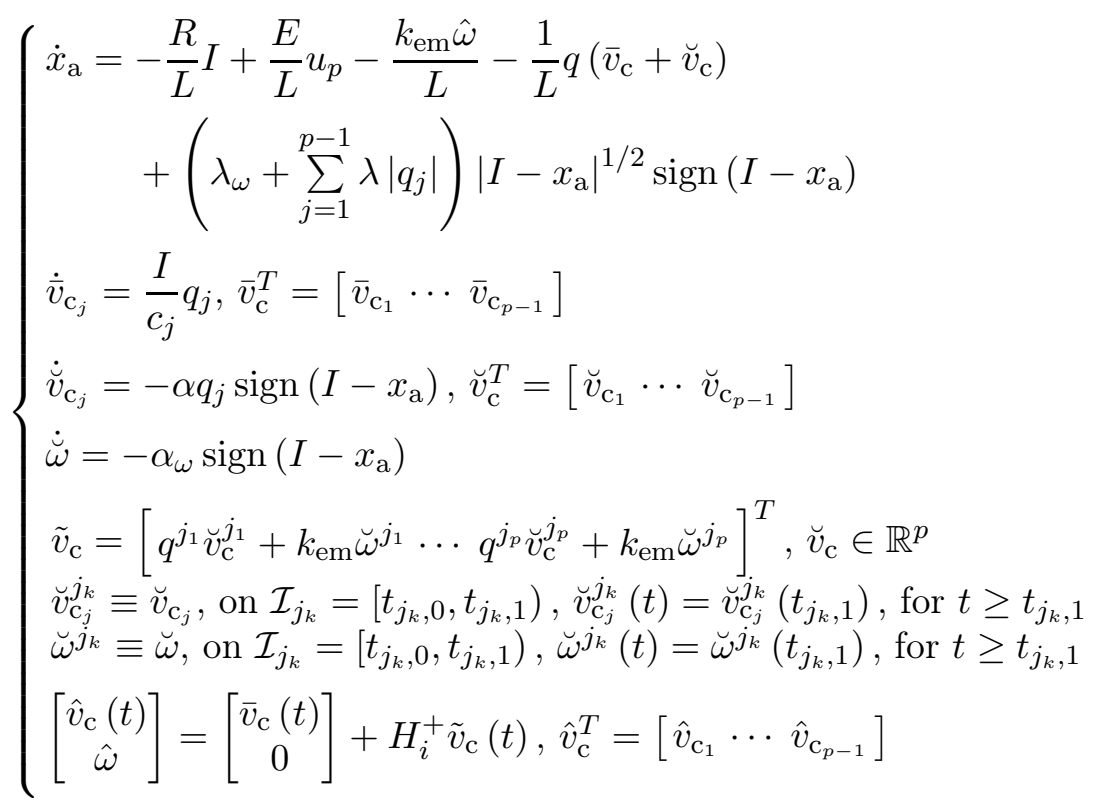

with $\alpha$ and $\lambda$ satisfying

$$
\begin{gathered}
\alpha>k \omega^{+}, \lambda>\frac{(1+\theta)\left(\alpha+k \omega^{+}\right)}{1-\theta} \sqrt{\frac{2}{L\left(\alpha-k \omega^{+}\right)}}, 0<\theta<1 \\
\alpha_{\omega}>\omega^{+}, \lambda_{\omega}>\frac{(1+\theta)\left(\alpha_{\omega}+\omega^{+}\right)}{1-\theta} \sqrt{\frac{2 k}{L\left(\alpha_{\omega}-\omega^{+}\right)}}, 0<\theta<1
\end{gathered}
$$

Defining $e_{1}=I-x_{\mathrm{a}}$, and $e_{\omega}=\omega-\hat{\omega}$ we obtain the following equations

$$
\begin{gathered}
\dot{e}_{1}=-k_{\mathrm{em}} e_{\omega}-\frac{1}{L} q\left(v_{\mathrm{c}}-\bar{v}_{\mathrm{c}}-\breve{v}_{\mathrm{c}}\right) \\
-e_{1}\left(\lambda_{\omega}+\sum_{j=1}^{p-1} \lambda\left|q_{j}\right|\right)\left|e_{1}\right|^{1 / 2} \operatorname{sign}\left(e_{1}\right) \\
\frac{d}{d t} q\left(v_{\mathrm{c}}-\bar{v}_{\mathrm{c}}+\breve{v}_{\mathrm{c}}\right)=-\alpha(p-1) \operatorname{sign}\left(e_{1}\right) \\
\dot{e}_{\omega}=\dot{\omega}+\alpha_{\omega} \operatorname{sign}\left(e_{1}\right)
\end{gathered}
$$

Thus, according to Levant (1996) and Davila et al. (2005), it exists a time $T_{i}>t_{i, 0}$ such that $e_{1}(t)=0, \dot{e}_{1}(t)=0$ for $t \geq T_{i}$. The reaching time to the sliding mode can be deduced from the proof of converge given in Davila et al. (2005), indeed,

$$
T_{i}-t_{i, 0} \leq \frac{1+\theta}{2 \theta\left(k \alpha_{\omega}+\alpha-k \omega^{+}\right)}\left(\sum_{j=1}^{p-1}\left|v_{c_{j}}(0)-\bar{v}_{c_{j}}(0)\right|+k|\omega(0)|\right)
$$

Thus, $\alpha_{\omega}$ and $\alpha$ must be chosen sufficiently big to satisfy $T_{i}-t_{i, 0}<\tau$ and in this way ensure $T_{i}<t_{i, 1}$. 
Thus, from the equation $\dot{e}_{1}$, we deduce

$$
q\left(v_{\mathrm{c}}-\bar{v}_{\mathrm{c}}-\breve{v}_{\mathrm{c}}\right)+k_{\mathrm{em}} e_{\omega}=0
$$

Theorem 5.4: Under assumptions $A 4, A 5$ and $\dot{\omega}=0$, the identities

$$
\begin{aligned}
\hat{v}_{\mathrm{c}_{i}} & \equiv v_{\mathrm{c}_{i}}, \forall i=1, \ldots, p-1 \\
\hat{\omega} & \equiv \omega
\end{aligned}
$$

are achieved for all $t>T_{p}$.

Proof The proof of theorem 5.4 can be followed likewise the proof of theorem 5.1; only it must be replaced the algorithm 1, Assumption A4 and Lemma 4.2 for the algorithm 2, Assumption A3 and lemma 4.4, respectively.

5.2.1.1 Error estimation for $\dot{\boldsymbol{\omega}} \neq \mathbf{0}$. In the general situation the speed of the motor is not required to be constant; in that case some consideration must be taken into account in order to have an acceptable estimation of the capacitors' voltages and, at the same time, of the motor speed. Indeed, let us find the error between the original and the estimated variables.

From the fact that the difference $q v_{\mathrm{c}}-q \bar{v}_{\mathrm{c}}$ is constant for any $q$, the following equalities are achieved for all $t>T_{i_{p}}$ :

$$
\begin{gathered}
q^{i_{p}} \bar{v}_{\mathrm{c}}(t)+q^{i_{p}} \breve{v}_{\mathrm{c}}^{i_{p}}=q^{i_{p}} v_{\mathrm{c}}(t)+k_{\mathrm{em}} \omega(t)+k_{\mathrm{em}}\left(\omega^{i_{p}}-\omega(t)\right) \\
\vdots \\
q^{i_{1}} \bar{v}_{\mathrm{c}}(t)+q^{i_{1}} \breve{v}_{\mathrm{c}}^{i_{1}}=q^{i_{1}} v_{\mathrm{c}}(t)+k_{\mathrm{em}} \omega(t)+k_{\mathrm{em}}\left(\omega^{i_{1}}-\omega(t)\right)
\end{gathered}
$$

By arranging the previous equalities in a matrix form, one gets

$$
\left[\begin{array}{c}
\bar{v}_{\mathrm{c}} \\
0
\end{array}\right]+H_{i}^{+} \tilde{v}_{\mathrm{c}}-k_{\mathrm{em}} H_{i}^{+}\left[\begin{array}{c}
\omega^{i_{p}}=i \\
\vdots \\
\vdots \\
\omega^{i_{1}}-\omega(t)
\end{array}\right]=\left[\begin{array}{c}
v_{\mathrm{c}} \\
\omega
\end{array}\right]
$$

Thus the error $e(t):=\left[\begin{array}{c}v_{\mathrm{c}} \\ \omega\end{array}\right]-\left[\begin{array}{c}\hat{v}_{\mathrm{c}} \\ \hat{\omega}\end{array}\right]$ takes the form

$$
e(t)=k_{\mathrm{em}} H_{i_{p}}^{+}\left[\begin{array}{c}
\omega^{i_{p}}-\omega(t) \\
\vdots \\
\omega^{i_{1}}-\omega(t)
\end{array}\right] \text { for } t_{i_{p}}>t \geq T_{i_{p}}
$$

Form the assumption A5, the error $\omega(t)-\omega\left(t_{i_{k}, 1}\right)$ can be estimated as it follows

$$
\left|\omega(t)-\omega\left(t_{i, 1}\right)\right|=\left|\int_{\tau=t_{i, 1}}^{t} \dot{\omega}(\tau) d \tau\right| \leq \omega^{+}\left(t-t_{i, 1}\right)
$$

Since $t_{i_{p}, 1}-t_{i_{1}, 1}>t_{i_{p}, 1}-t_{i_{k}, 1} \geq t-t_{i_{k}, 1}$, for $p-1 \geq k \geq 2$ and $t \in\left[T_{i}, t_{i, 1}\right)$, one can say that the estimation error $e(t)$ on the interval $\mathcal{I}_{i_{p}}$ is $O\left(t_{i_{p}, 1}-t_{i_{1}, 1}\right)$. This can be summarized by the following theorem. 
Theorem 5.5: With the observer given by (29), the estimation error e $(t)$ defined in (13), for $t \in\left[T_{i_{p}}, t_{i_{p}, 1}\right)$, has a bounded norm, whose upper-bound is given by:

$$
\|e(t)\| \leq p k_{\mathrm{em}} \omega^{+}\left\|H_{i_{p}}^{+}\right\|\left|t_{i_{p}, 1}-t_{i_{1}, 1}\right|
$$

where $\|\dot{\omega}(t)\| \leq \omega^{+}$.

Notice that for the case $\dot{\omega}(t)=0$, theorems 5.4 and 5.5 coincide.

In the practical case, the relation $t_{i_{p}, 1}-t_{i_{1}, 1}<<1$ is not difficult to be fulfilled since the dwell time between each commutation might be very short, which may assure an acceptable small estimation error.

\subsubsection{Partial state reconstruction}

There could be the case when the vector $\left[x^{T} \omega\right]^{T}$ is not $Z\left(T_{N}\right)$-observable, but it could still be interesting to observer only a set of variables. Here we give a general form for the design of an observer for such a case. For simplicity, let us define $v_{\mathrm{c}_{p}}:=\omega$. Hence, define the vector $z \in \mathbb{R}^{r}$ as $z:=\left[\bar{z}_{1} \cdots \bar{z}_{r}\right]^{T}$ with $r \leq p$, where the terms of $z$ are such that $\bar{z}_{j} \in\left\{v_{\mathrm{c}_{1}}, \ldots, v_{\mathrm{c}_{p}}\right\}$ for $j=1, \ldots, r$. The current load is not included in the vector $z$ since it is already known.

In order to be able to estimate the vector $z$, we need to make an assumption that guaranties the observability of the vector $z$, and, at the same time, that allows the design of an observer. Let $T \in \mathbb{R}^{p \times r}$ be a matrix whose $j$-th column has zeros everywhere except that it has 1 in the $h$-th row satisfying ${ }^{1} v_{\mathrm{c}_{h}}=\bar{z}_{j}$, for $j=1, \ldots, r$. It should be noticed that $T^{T}\left[x^{T} \omega\right]^{T}=z$ Now, let us assume that:

A4(b). It exists a vector sequence $\left\{q^{i_{j}}\right\} \quad(j=1, \ldots, p)$ so that the span of $\left\{\left[\begin{array}{ll}q^{i_{1}} & 1\end{array}\right]^{T}, \ldots,\left[q^{i_{p}} 1\right]^{T}\right\}$ contains the subspace spanned by the columns of $T$.

For the case $\dot{\omega}=0$, it can be verified that the condition $\mathrm{A} 4(\mathrm{~b})$ is equivalent to say that the vector $z$ is $Z\left(T_{N}\right)$-observable.

Now, let $H_{i}$ is given by (2), Notice that the A4(b) also implies the existence of a matrix $G_{i}$ accomplishing the identity $G_{i} H_{i}=T^{T}$, for $i \geq i_{p}$.

Thus, the observer $\hat{z}$ is designed by means of

$$
\hat{z}(t):=G_{i} H_{i}\left[\begin{array}{c}
\bar{v}_{\mathrm{c}}(t) \\
0
\end{array}\right]+G_{i} \tilde{v}_{\mathrm{c}}(t)
$$

where $\bar{v}_{\mathrm{c}}$ and $\tilde{v}_{\mathrm{c}}$ are defined according to (29).

The next theorem claims the reconstruction of the $z$ by means of $\hat{z}(t)$.

Theorem 5.6: Assuming that the conditions A4(b), A5 and $\dot{\omega}=0$ are fulfilled, the identity

$$
\hat{z}(t) \equiv z(t)
$$

is achieved for all $t>T_{i_{p}}$.

Proof From the arguments of the proof of theorems 5.1 and 5.4, we have:

$$
H_{i}\left[\begin{array}{c}
\bar{v}_{\mathrm{c}}(t) \\
0
\end{array}\right]+\tilde{v}_{\mathrm{c}}(t)=H_{i}\left[\begin{array}{l}
v_{\mathrm{c}}(t) \\
\omega(t)
\end{array}\right]
$$

\footnotetext{
${ }^{1}$ For instance, if $p=4$, and $z=\left[\begin{array}{ll}v_{\mathrm{c}_{1}} & v_{\mathrm{c}_{4}}\end{array}\right]^{T}$, then $T^{T}=\left[\begin{array}{llll}1 & 0 & 0 & 0 \\ 0 & 0 & 0 & 1\end{array}\right]$.
} 
Therefore, because of $T^{T}\left[v_{\mathrm{c}}^{T} \omega\right]^{T}=z$ and $G_{i} H_{i}=T^{T}$ for $i \geq i_{p}$, the last identity yields

$$
G_{i} H_{i}\left[\begin{array}{c}
\bar{v}_{\mathrm{c}}(t) \\
0
\end{array}\right]+G_{i} \tilde{v}_{\mathrm{c}}(t)=z
$$

Thus, comparing (33) and (35), we obtain (34).

Remark 4: For covering the case $\dot{\omega}(t) \neq 0$, an estimation error could be computed . This can be done in the same way as in 5.2.1.1, obtaining quite similar results.

It is clear that the partial observation, with the observer (33), includes the full state observation, with the observer (29); however, since the main purpose of this paper is the design of full state observers, we have preferred to explain with more details the full state observation.

\subsubsection{Description of the Adaptive Observer}

By using $H_{i}$ designed according to the algorithm 2, the adaptive observer estimating the voltages in the capacitors and the motor speed by using only the current measurement is designed by the following set of equations

$$
\left\{\begin{array}{l}
\dot{Z}=A Z+H(\tilde{Z})+G(u, y)+P^{-1} C^{T}(y-\hat{y}) \\
\dot{P}=-\rho P-A^{T} P-P A+2 C^{T} C \\
\hat{y}=C Z \\
\dot{\tilde{Z}}=\tilde{A} \tilde{Z}+\tilde{H}(Z)+G(u, y) \tilde{P}^{-1} C^{T}(y-\hat{\tilde{y}}) \\
\dot{\tilde{P}}=-\tilde{\rho} \tilde{P}-\tilde{A}^{T} \tilde{P}-\tilde{P} \tilde{A}+2 C^{T} C \\
\hat{\tilde{y}}=C \tilde{Z} \\
\dot{\bar{v}}_{\mathrm{c}_{j}}=\frac{q_{j}}{c_{j}} I ; \quad \bar{v}_{\mathrm{c}}=\left[\bar{v}_{\mathrm{c}_{1}} \cdots \bar{v}_{\mathrm{c}_{p-1}}\right]^{T} \\
\Lambda=\hat{b}+k_{\mathrm{em}} \tilde{\omega}-q \bar{v}_{\mathrm{c}} \\
\tilde{v}_{\mathrm{c}}=\left[\Lambda^{i_{p}} \cdots \Lambda^{i_{1}}\right]^{T} \\
\Lambda^{i_{k}} \equiv \Lambda, \text { on } \mathcal{I}_{i_{k}}=\left[t_{i_{k}, 0}, t_{i_{k}, 1}\right), \Lambda^{i_{k}}(t)=\Lambda\left(t_{i_{k}, 1}\right), \text { for } t \geq t_{i_{k}, 1} \\
{\left[\begin{array}{c}
\hat{v}_{\mathrm{c}}(t) \\
\hat{\omega}
\end{array}\right]=\left[\begin{array}{c}
\bar{v}_{\mathrm{c}}(t) \\
0
\end{array}\right]+H_{i}^{+} \tilde{v}_{\mathrm{c}}(t)} \\
\end{array}\right.
$$

where $Z^{T}=\left[\begin{array}{ll}\hat{I} & \hat{b}\end{array}\right]$ and $A, G(u, y), C$ are defined in $(20) . \tilde{Z}^{T}=\left[\begin{array}{cc}\tilde{I} & \tilde{w}\end{array}\right], \tilde{A}=\left[\begin{array}{cc}0 & -\frac{k_{e m}}{L} \\ 0 & 0\end{array}\right] . H(\tilde{Z})=$ $\left[-\frac{k_{e m}}{L} \tilde{w} 0\right]^{T}$ and $\tilde{H}(Z)=\left[-\frac{1}{L} \hat{b} 0\right]^{T} \cdot \rho>0$ and $\tilde{\rho}>0$.

5.2.3.1 Convergence analysis. Defining the estimation errors $e=X-Z$ and $\tilde{e}=\tilde{X}-\tilde{Z}$. Their dynamics are given by

$$
\left\{\begin{array}{l}
\dot{e}=\left[A-P^{-1} C^{T} C\right] e+[H(\tilde{X})-H(\tilde{Z})] \\
\dot{\tilde{e}}=\left[\tilde{A}-\tilde{P}^{-1} C^{T} C\right] \tilde{e}+[\tilde{H}(X)-\tilde{H}(Z)]
\end{array}\right.
$$

Consider now the Lyapunov function candidate $V=V(e)+V(\tilde{e})=e^{T} P e+\tilde{e}^{T} \tilde{P} \tilde{e}$ and taking the time derivative of $V$ along the trajectory (37), it follows that

$$
\dot{V}=-\rho V(e)-\tilde{\rho} V(\tilde{e})+2 e^{T} P[H(\tilde{X})-H(\tilde{Z})]+2 \tilde{e}^{T} \tilde{P}[\tilde{H}(X)-\tilde{H}(Z)]
$$

By taking the norm, one has

$$
\dot{V} \leq-\rho V(e)-\tilde{\rho} V(\tilde{e})+2 K\|e\|\|\tilde{e}\|+2 \tilde{K}\|\tilde{e}\|\|e\|
$$


where $K=\frac{k_{\mathrm{em}}}{L}$ and $\tilde{K}=\frac{1}{L}$.

Now, consider that the following inequalities are satisfied

$$
\begin{aligned}
& \lambda_{1}\|e\|^{2} \leq\|e\|_{P}^{2} \leq \lambda_{2}\|e\|^{2}, \quad\|e\|_{P}^{2}=e^{T} P e \\
& \tilde{\lambda}_{1}\|\tilde{e}\|^{2} \leq\|\tilde{e}\|_{\tilde{P}}^{2} \leq \tilde{\lambda}_{2}\|\tilde{e}\|^{2}, \quad\|\tilde{e}\|_{\tilde{P}}^{2}=\tilde{e}^{T} \tilde{P} \tilde{e} .
\end{aligned}
$$

By writing (38) in terms of functions $V_{1}$ and $V_{2}$, it follows that

$$
\dot{V} \leq-\rho V(e)-\tilde{\rho} V(\tilde{e})+2\left(\mu_{1}+\mu_{2}\right) \sqrt{V(e)} \sqrt{V(\tilde{e})}
$$

where the parameters $\mu_{1}=\frac{2 K}{\lambda_{1} \tilde{\lambda}_{1}}, \mu_{2}=\frac{2 \tilde{K}}{\lambda_{1} \tilde{\lambda}_{1}}$.

Next, by using the following inequality $\sqrt{V(e)} \sqrt{V(\tilde{e})} \leq \frac{\epsilon}{2} V(e)+\frac{1}{2 \epsilon} V(\tilde{e}), \forall \epsilon \in(0,1)$, one gets

$$
\dot{V} \leq-\rho V(e)+\left(\mu_{1}+\mu_{2}\right) \epsilon V(e)-\tilde{\rho} V(\tilde{e})+\frac{\left(\mu_{1}+\mu_{2}\right)}{\epsilon} V_{2}
$$

Finally, defining:

$$
\theta_{1}=\rho-\left(\mu_{1}+\mu_{2}\right) \epsilon>0
$$

and

$$
\theta_{2}=\tilde{\rho}-\frac{\left(\mu_{1}+\mu_{2}\right)}{\epsilon}>0
$$

where $\rho$ and $\tilde{\rho}$ are chosen such that the inequalities (39) and (40) are satisfied. Then by choosing $\theta$ such that $\theta=\min \left(\theta_{1}, \theta_{2}\right)$ it follows that

$$
\dot{V} \leq-\theta(V(e)+V(\tilde{e})) \leq-\delta V
$$

According to (41) and following the same procedure in it exists a time $T_{i} \leq t_{i, 0}$ such that

$$
\|e(t)\|+\|\tilde{e}(t)\| \leq \Delta, \forall t \geq t_{i, 1}
$$

where $\Delta$ is an acceptable constant smaller error after convergence. Thus, $\rho$ and $\tilde{\rho}$ must be chosen sufficiently large and according to the equalities (39) and (40) in order to satisfy $T_{i}-t_{i, 0}<\tau$ and to ensure in this way $T_{i} \leq t_{i, 0}$. Then from (42) and equation of $\Lambda$ in (36), the following can be deduced

$$
\Lambda^{j_{k}}+q \bar{v}_{\mathrm{c}}=q v_{\mathrm{c}}+k_{\mathrm{em}} \omega+\varphi^{j_{k}}
$$

where $\varphi^{j_{k}}=\hat{b}\left(t_{j_{k}, 1}\right)-b\left(t_{j_{k}, 1}\right)+k_{\mathrm{em}}(\hat{\omega}-\omega)$ is a small enough constant.

Theorem 5.7: Under assumptions A4, A5, and $\dot{\omega}=0$, the identities

$$
\left[\begin{array}{c}
\hat{v}_{c} \\
\hat{\omega}
\end{array}\right]=\left[\begin{array}{l}
v_{c} \\
\omega
\end{array}\right]+H_{i}^{+} \varphi
$$

are achieved for all $t \in t \in\left[t_{i_{p}, 1}, t_{N}\right]$ where $\varphi=\left[\varphi^{i_{p}} \cdots \varphi^{i_{1}}\right]^{T}$.

Proof The theorem can been proven by replacing in the proof of theorem 5.3, A3 and Lemma 4.4 by $\mathrm{A} 4$ and Lemma 4.2, respectively. 


\subsubsection{Simulations for a 3 cells chopper}

Here, we consider the 3 cells chopper given by (28) with a DC motor as load with the $k_{\mathrm{em}}=$ $\frac{100}{157}(\mathrm{rad} / \mathrm{s})^{-1} \mathrm{~V}$. Thus, the state equations take the form

$$
\left\{\begin{aligned}
\dot{I}(t) & =-6600 I(t)+2400 u_{3}(t)-20\left(q_{1} v_{\mathrm{c}_{1}}(t)+q_{2} v_{\mathrm{c}_{2}}(t)\right)-\frac{2000}{157} \omega(t) \\
\dot{v}_{\mathrm{c}_{1}}(t) & =q_{1} \frac{10^{6}}{40} I(t) \\
\dot{v}_{\mathrm{c}_{2}}(t) & =q_{2} \frac{10^{6}}{40} I(t) \\
y(t) & =I(t)
\end{aligned}\right.
$$

where the same PWM described in section (5.1.3) is adopted for the control of switches.

In this case, the motor speed is supposed to be given by $\omega(t)=2 \sin (4 \pi t+1)+2$. The parameters of the STO are $\alpha=10000, \alpha_{\omega}=1000, \lambda=3300$ and $\lambda_{\omega}=330$. The parameters $\rho$ and $\tilde{\rho}$ of the $\mathrm{AO}$ are chosen as $\rho=1500$ and $\tilde{\rho}=1200$.

Here, the motor speed varies slow with respect to the frequency of the switching control; indeed, we have $\left|\omega\left(t_{2}\right)-\omega\left(t_{1}\right)\right| \leq 8 \pi\left|t_{2}-t_{1}\right|$ and the switch time is $\tau \simeq 3 \times 10^{-4}$. Let $\varkappa$ be the number of switchings needed to fulfilled the $Z\left(T_{N}\right)$-observability(Lemma 4.2), then, from theorem 5.5, $\|e(t)\| \leq 0.014 \varkappa\left\|H_{i}^{-1}\right\|$ for $t \geq T_{\varkappa}$. Figure 4 shows the voltage and its estimation in the first and second capacitor. The motor speed and its estimation are shown in figure 5 . In spite of having a non constant speed the estimation errors are acceptably small.

As we proved, in that case, the estimation error is proportional to the dwell time and the maximal velocity. Thus, as we can see in both figures, the estimation can be done with a very small error despite the system is not observable (neither in the classical sense nor according to $Z\left(T_{N}\right)$-observability definition).
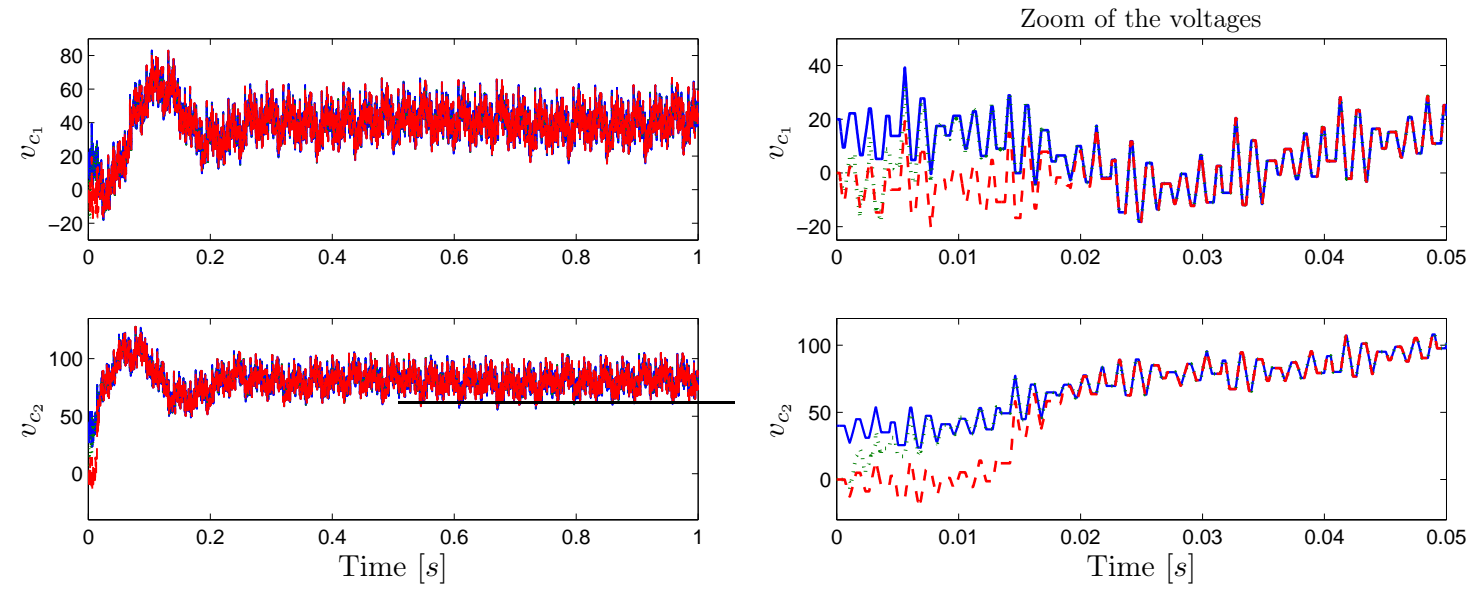

Figure 4. $v_{\mathrm{c}_{\mathrm{i}}}$ (solid) $(\mathrm{i}=1,2)$ and $\hat{v}_{\mathrm{c}_{\mathrm{i}}}$, STO (dot) and AO (dashed). 

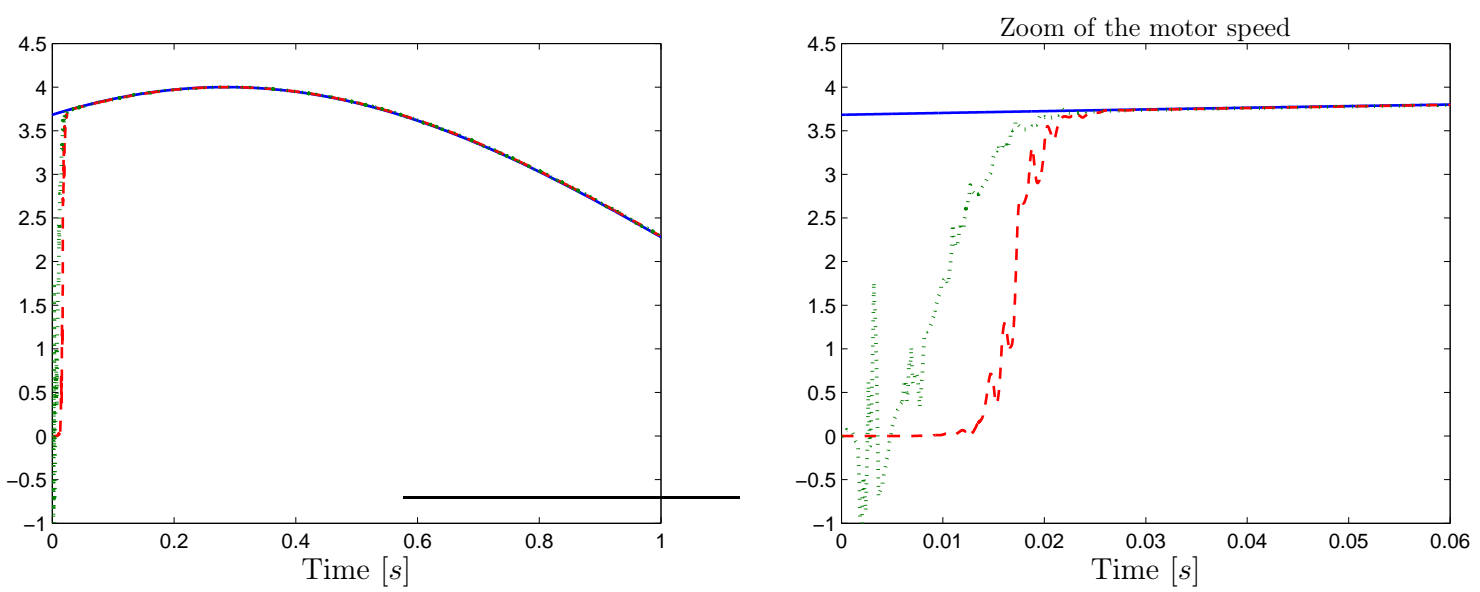

Figure 5. $\omega$ (solid) and $\hat{\omega}$, STO (dot) and AO (dashed).

Some simulations were made in order to test the robustness of the considered observers with respect to noise in the system output. That is, the output of the system is given by $y(t)=$ $I(t)+\xi(t)$, where $\xi(t)$ represents the noise. The function $\xi(t)$ used in the simulations is depicted in figure 6 . The voltage estimation is presented in figure 7 ; there we can see that the observers are rather robust and the effect of the noise is almost imperceptible. In opposition, as we see in figure 8 , the motor speed estimation seems to be more sensitive to noise in the system output. Here it is important to say that for the case of the STO, the super-twisting works like a numerical differentiator and for this exists a result giving an estimate of the robustness of this differentiator with respect to noise (see Levant (1996)).

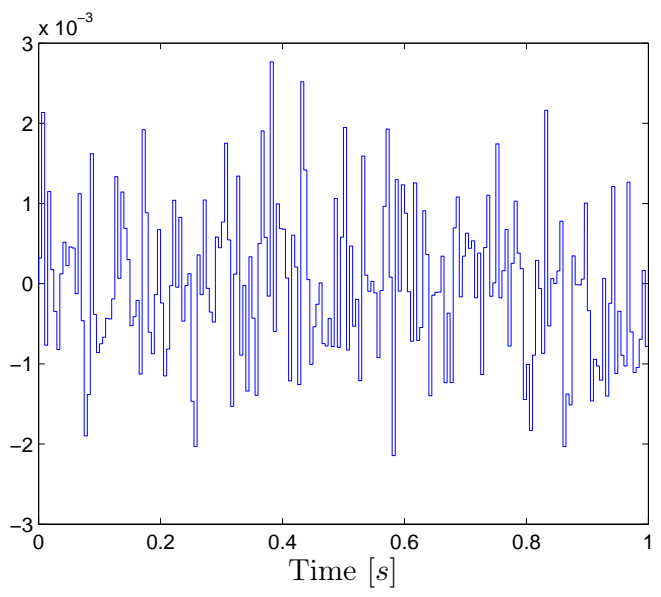

Figure 6. Noise $\xi$ affecting the system output.
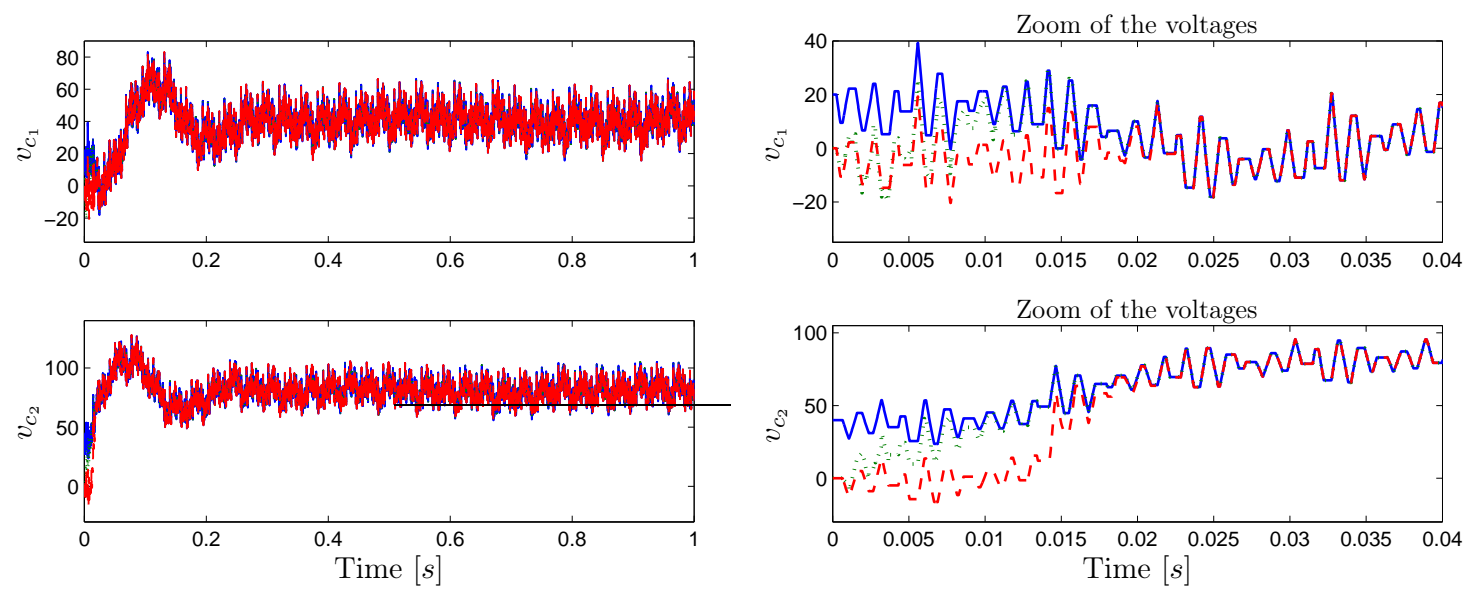

Figure 7. $v_{\mathrm{C}_{\mathrm{i}}}$ (solid) $(\mathrm{i}=1,2)$ and $\hat{v}_{\mathrm{C}_{\mathrm{i}}}$, $\mathrm{STO}$ (dot) and $\mathrm{AO}$ (dashed), when the output is affected by noise. 

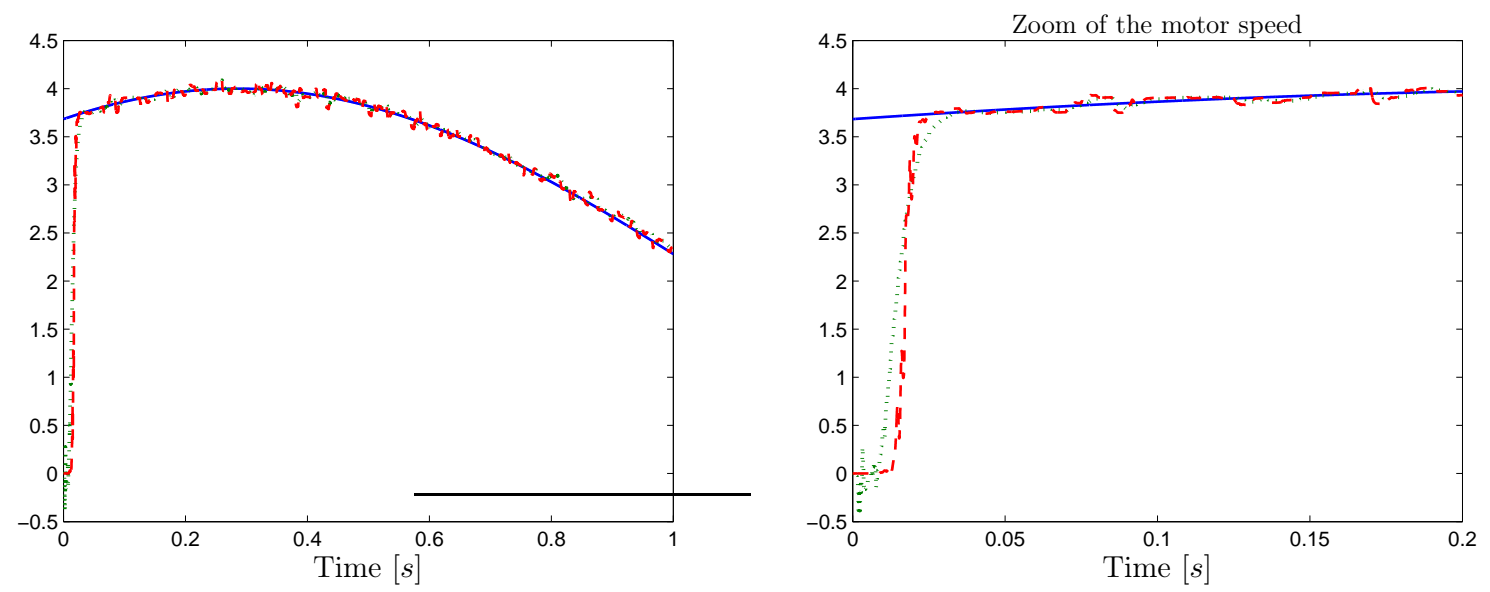

Figure 8. $\omega$ (solid) and $\hat{\omega}$, STO (dot) and AO (dashed), when the output is affected by noise.

\section{Conclusions}

In this manuscript we consider the multicell-choppers belonging to a sort of hybrid systems. To observer the voltages across the capacitors, the system needs to be $Z\left(T_{N}\right)$-observable. It was shown that even when, on an interval of time when all switches do not change its position (the control stays constant), the system is non-observable in the classical sense, it is still possible to estimate the voltage on every capacitor after a switching control sequence. That is, theoretically the voltages on the capacitors can not be estimated instantaneously after any time greater than zero (observability in the classical sense), but they can be estimated after some time by saving the information recovered before a switching occurs.

Furthermore, it has been shown than the speed of a DC-motor coupled to the multicell can be estimated with an acceptable error due to the fact that the dynamics of the electrical part (multicell chopper) is much faster than the one of the mechanical part (DC-motor). Some additional results show an observer for the partial state observation and the conditions under which it can be designed.

It must be noticed that to assure the convergence of both observers to the original values, it was needed to compute the convergence time, which depends on the initial conditions of the original state vector. Therefore, it must be said that the convergence of both observers, STO and $\mathrm{AO}$, is semi-global.

\section{References}

Besançon G, De Leon-Morales J, et al. On adaptive observers for state affine systems. International Journal of Control 2006; 79(6):581-591.

Bensaid R, Fadel M, and Meynard T. Observer design for a three cells chopper using discrete-time model. Electromotion 1999; 2:689-694.

Bensaid R and Fadel M. Sliding modes observer for multicell converters. IFAC NOLCOS, St. Petersburg, Russia, 2001.

Bethoux $\mathrm{O}$ and Barbot J-P. Multi-cell chopper direct control law preserving optimal limit cycles. IEEE CCA, vol. 2, Glasgow, 2002; 1258-1263.

Daafouz J, Rieding P, and Iung C. Stability Analysis and Control Synthesis for Switched Systems. IEEE Transactions on Automatic Control 2002; 47:1883-1887.

Davila J, Fridman L, and Levant A. Second-order sliding-mode observer for mechanical systems. IEEE Transactions on Automatic Control 2005; 50(11):1785-1789 . 
Donzel A. Commande des convertisseurs multiniveaux: application à un moteur asynchrone. Thèse de doctorat, Institut National Polytechnique de Grenoble, 2002.

Erickson R and Maksimovic D. Fundamentals of Power Electronics. Kluwer Academic Publishers: Dordrecht, The Netherlands, 2001.

Fadel M and Meynard TA. Equilibrage des tensions dans les convertisseurs statiques multicellulaires série: modélisation. EPF, Grenoble, 1996; 115-120.

Gateau G, Fadel M, Maussion P, Bensaid R, and Meynard TA. Multicell converters: active control and observation of flying-capacitor voltages Industrial Electronics. IEEE Transactions on Automatic Control 2002; 49(5):998-1008.

Gauthier J-P and Kupka I. Deterministic observation theory and applications. Cambridge University Press, 2001.

Ghanes M, De Leon J, and Glumineau A. Observability study and observer-based interconnected form for sensorless induction motor. Proc 45th IEEE Conference on Decision and Control, San Diego, CA, 2006.

Goiebel R, Hespanha J, Teel AR, Cai C, and Sanfelice R. Hybrid systems: generalized solutions and robust stability. IFAC NOLCOS, 2004.

Hammouri $\mathrm{H}$ and De Leon J. Observer synthesis for state-affine systems, Proc 29th IEEE Conference on Decision and Control, Honolulu, Hawaii. 1990.

Kang W and Barbot J-P. Discussions on Observability and invertibility, IFAC NOLCOS, 2007.

Levant A. Sliding order and sliding accuracy in sliding mode control. International Journal of Control 1996; 58(6):1247-1263.

Lygeros J, Johansson HK, Sinć SN, Zhang J, Sastry SS. Dynamical properties of hybrid automata. IEEE Transactions on Automatic Control 2003; 48(1):2-17.

Tachon O, Fadel M, Meynard T. Control of series multicell converters by linear state feedback decoupling. EPF, Grenoble, 1997; 1588-1593. 